\title{
Toward developing a tele-diagnosis system on fish disease
}

\author{
Daoliang $\mathrm{Li}^{1 *}$, Wei $\mathrm{Zhu}^{1}$, Yanqing Duan ${ }^{2}$, Zetian $\mathrm{Fu}^{1}$ \\ 1 Key Laboratory of Modern Precision Agriculture system \\ Integration, P.O. Box 121, China Agricultural University, Beijing, \\ 100083 , P. R. China \\ 2 Luton Business School, University of Luton, LU1 3JU, UK
}

\begin{abstract}
Fish disease diagnosis is a complicated process and requires high level of expertise, an expert system for fish disease diagnosis is considered as an effective tool to help fish farmers. However, many farmers have no computers and are not able to access the Internet. Telephone and mobile uses increase rapidly, so, the provision of call centre service appears as a sound alternative support channel for farmer to acquire counseling and support. This paper presents a research attempt to develop and evaluate a call center oriented Hybrid disease diagnosis \& consulting system (H-Vet) in aquaculture in China. This paper looks at why H-Vet is needed and what are the advantages and difficulties in the developing and using such a system. A machine learning approach is adopted, which helps to acquire knowledge when enhancing expert systems with the user information collected through call center. This paper also proposes a fuzzy Group Support Systems (GSS) framework for acquiring knowledge from individual expert and aggregating knowledge into workgroup knowledge by $\mathrm{H}-\mathrm{Vet}$ in the situation of difficult disease diagnosis. The system's architecture and components are described.
\end{abstract}

Keywords machine learning; Group Decision Support System, expert system, call centre

\section{Introduction}

In China, Aquaculture plays a very important role in agricultural structure adjustment and generating farmers' income (Guo, 2001). However, fish diseases have become one of the most devastating threats to the survival of many Chinese fish farms. Fish disease diagnosis is a complicated process and requires high level of

* Corresponding author. Tel: +86-10-62336717; Fax:+86-10-62324371.

Email address: li_daoliang@yahoo.com or dliangl@cau.edu.cn (D. Li)

Please use the following format when citing this chapter:

Li, D., Zhu, W., Duan, Y., Fu, Z., 2006, in IFIP International Federation for Information Processing, Volume 217, Artificial Intelligence in Theory and Practice, ed. M. Bramer, (Boston: Springer), pp. 445-454. 
domain knowledge, this pose a major challenge for any attempts to provide accurate and timely diagnosis and treatment.

Advances of Internet technologies have offered new opportunities for enhancing traditional decision support systems and expert systems (Power, 2000). With the development of Expert Systems (ES) and multimedia, computers are able to mimic many important roles that normally require human actions. A number of Web-based expert systems are reported in the literature (Grove, 2000; Potter et al., 2000; Riva, Bellazi, \& Montani, 1998; Sedbrook, 1998).

Developments in high performance global communication technologies have also accelerated cooperative image-based medical services to a new frontier. Traditional image-based medical services such as radiology and diagnostic consultation can now be fully enhanced by multimedia technologies to provide novel services, such as tele-medicine. Telemedicine can be defined as medical practices across distance via telecommunications and interactive video technology. It can be used in remote areas or across great distances on the globe (Lim, Pang, \& Tan, 2001). It also covers any form of communication between health workers and patients through electronic equipment from remote locations. Similar to tele-medicine, a telediagnosis system has been developed to support the diagnosis of various types of problems in China (Duan, et al.,2003), which provide a farmer-to-vet communication in diagnosis.

However, many farmers have no computers and are not able to access the Internet. At the same time, telephone and mobile uses increase rapidly, especially in rural area of China, there are 0.24 billion telephone users, $40 \%$ of them are farmers, so, the provision of call centre service appears as a sound alternative support channel for farmer to acquire counseling and support in disease diagnosis in China.

This paper reports a research effort to integrate call center with web-based expert system and tele-diagnosis system in fresh water fish disease diagnosis in China. The system is being developed by Key Laboratory of Modern Precision Agriculture System Integration, China Agricultural University, and was funded by Huo Yingdong foundation in China. The system has emerged as a result of the use of a Web-based expert system called Fish-Expert and tele-diagnosis system called TVet. It helps to overcome the limitations and enhance the functionality of traditional ESs. One of the strengths of the system is that it can facilitate computer-not-owned users and knowledge remote acquisition. This research attempts to use this pilot system as a research vehicle to experiment in applying, and to evaluate the usability of, the system with potential users. Feedback collected from the demonstration and evaluation of the Fish-Expert and T-vet has provided valuable insights into the issues related to the development and implementation of H-Vet in China.

\section{System architecture and components}

Based on the user's needs analysis, a call center oriented tele-diagnosis system, called CORDIS, were developed and integrated to Fish-Expert and T-Vet, H-Vet

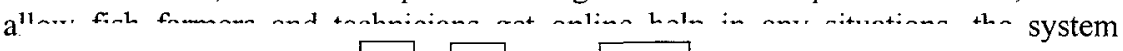
s1

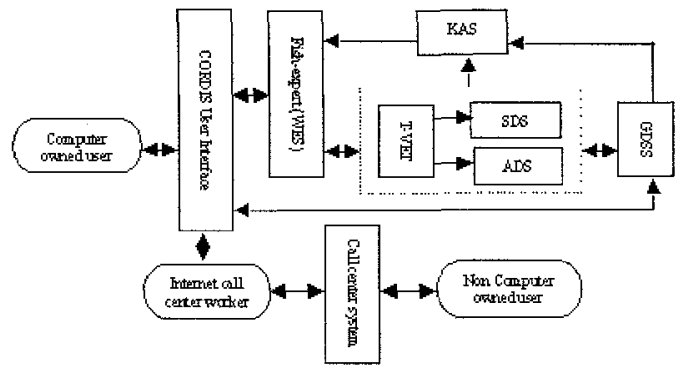


Fig. 1. Structure and Subsystem in H-Vet

To best meet the different needs of fish farmers and vets, 5 subsystems have been designed and developed in $\mathrm{H}$-vet, they are Web-based Expert System FishExpert (WES), Call Center System (CCS), Tele-diagnosis System (T-VET), Group Decision Support System (GDSS), and Knowledge Acquisition System (KAS).

As more details about web-based expert system Fish-Expert and tele-diagnosis system (T-VET) are described in $\mathrm{Li}$, et al. (2002) and Duan, et al. (2003), FishExpert and T-vet will be described very shortly. More contents will cover the CCS, GDSS, and KAS.

\subsection{Web-based expert system Fish-Expert (WES)}

The Fish-Expert system mimics the diagnosing process of human experts and has over 300 rules and 400 images and graphics for different types of diseases and symptoms. It is able to diagnose 126 types of diseases amongst nine species of primary freshwater fish. When using the expert system, various information needs to be provided following different diagnosing steps, such as pond inspection, fish inspection and anatomization, water quality examination, and microscopic examination. A final verdict for the type of the disease and its treatments and prevention will be produced based on the system's knowledge base.

\subsection{Tele-diagnosis system T-Vet}

T-vet is an add-on subsystem to overcome the limitation of the expert system and was integrated to the Fish-Expert. It includes a synchronous tele-diagnosis subsystem (SDS) and an asynchronous diagnosis subsystem (ADS) (Duan,et al., 2003)

A synchronous tele-diagnosis subsystem has been developed and can be used in situations where an urgent diagnosis is required, but it is impossible for the fish vet to visit the site, and the Web-based expert system is not able to solve the problem. To facilitate the tele-diagnosis, a number of functions have been developed and integrated into the system, such as web-calling, a virtual diagnosis room, Computer Supported Cooperative Work (CSCW) module, video/audio conferencing module, and online help module. An asynchronous diagnosis system (ADS) has been developed, which acts as a practical platform for sending and receiving messages between a fish farmer and a vet. Three support modules-user symptom submission, vet diagnosis and email communication are designed to facilitate asynchronous diagnosis process.

The ADS and the SDS act as good complementary tools to the expert system. The integration of the expert system, synchronous and asynchronous systems complements each other and is able to solve most of the problems fish farmers may encounter. 


\subsection{Call Center System (CCS)}

The Call Center System CSS is an add-on subsystem to overcome the limitation of the current low computer owned level. It can provide a bridge between the computernot-owned fish farmer and Fish-expert, T-Vet through call center agent,. There are 3 main models in the system, such as queuing models to capture the impact of congestion, customer arrival statistics and data collection model, telecommunications resource allocation and telephone-agent staffing model.

As call centers have grown in number and in size, more firms have tried to improve their management by focusing on resource utilization and service levels. This has led to a series of studies dealing with the problem of staffing phone centers, many of which have made use of queuing models to capture the impact of congestion (Aksin \& Harker, 2003). So queuing models should be designed to capture the impact of congestion.

Customer arrival statistics and corresponding data collection model is used to evaluate the performance of the service system under study, given customer arrival statistics, servers, buffers, and a shared resource that impacts processing times, to collect all disease case which will be used to acquire fish disease diagnosis knowledge.

Another model take a different approach to telecommunications resource allocation and telephone-agent staffing, a similar approach is taken to determine staffing levels for a multiple class inbound call center.

CCS not only provides a bridge between the computer-not-owned fish farmer and Fish-Expert, but also provides a tool for collecting fish disease diagnosis case which plays a very important role in Fish-expert.

\subsection{Group Decision support System (GDSS)}

Globalization, virtual corporations, telecommuting, empowerment of teams, reduced cycle time and the need to frequently make decisions quickly makes it necessary for groups to work together while the participants may be in different locations (Tung \& Turban, 1998). Distributed Group Decision Support System (DGSS) is a technology that can help groups to overcome some of the difficulties associated with being in different places and sometimes in different time zones (Bendoly \& Bachrach, 2003).

Fish disease diagnosis is a rather complicated process in aquaculture production activities. The disease commonly resulted from nutritional and environmental problems as well as infections by parasites, viruses, bacteria and fungal agents. Some rare diseases or new diseases normally can't be identified by one fish vet only, and most of them need to be diagnosed by group work, as result, a Group Decision Support System (GDSS) is essential needed to solve the rare disease diagnosis.

Most of the previous research regarding computer support of groups was related to the decision room environment, where a group of participants meet face-to-face, working on a common task (Stohr \& Konzynski, 1992). This paper focuses on the technology to support group work in the framework of 3 situations, such as sametime same-place group work, different-place same-time and different-time differentplace group work. 
Both same-time same-place group work and different-place same-time group work are belong to synchronous GDSSs, the same-time same-place group work is a face-to-face communication work mode for the decision support. This kind of group work has no any limitation of the work environment, the fish vet can discuss together, how ever the shortcoming of this kind of group work all famous vet must be collected together in call center, and answer the user's questions for some rare disease diagnosis.

Different-place same-time group work is synchronous GDSSs allow distributed participants to interact with one another in a 'real time' mode, i.e., they interact with one another at the same time. The participants are distributed across multiple sites linked by various communication technologies. Some of the supporting technologies are screen sharing, whiteboard, audio-conferencing, and various types of videoconferencing. These technologies can be carried on the Intranet, Internet, corporate or public networks, or VANs. Some major issues here include: the loss of face-toface contact and the ability to manage the group process.

Asynchronous DGSSs allow distributed participants to log into the same meeting but at different times. Participants can log in and catch up with what is going on in the meeting, enter comments if necessary, and log out of the meeting at various times. E-mail, voice-mail, and workflow management systems such as in Lotus Notes are some of the supporting technologies. The issues here are more than just time and distance barriers. For example, the control of the participants and the participation, and the delayed response time could play an important role. Therefore, attention must be given to the coordination of group members to ensure that they stay on task and track and meet the decision deadlines, as well as it is necessary to encourage timely participation by everyone.

As the rare fish disease cases are very short in the fish disease case base of the web-based expert system, all result of group decision will be acquired by the KAS.

\subsection{Knowledge Acquisition System (KAS)}

Call center system, Fish-expert, T-vet, and GDSS can provide plenty of successful fish disease cases and solutions, these cases and solutions are very important for the Fish-Expert, so how to integrate them together and make them cooperate together, and then get a best effect for the whole system poses a serious challenge. 


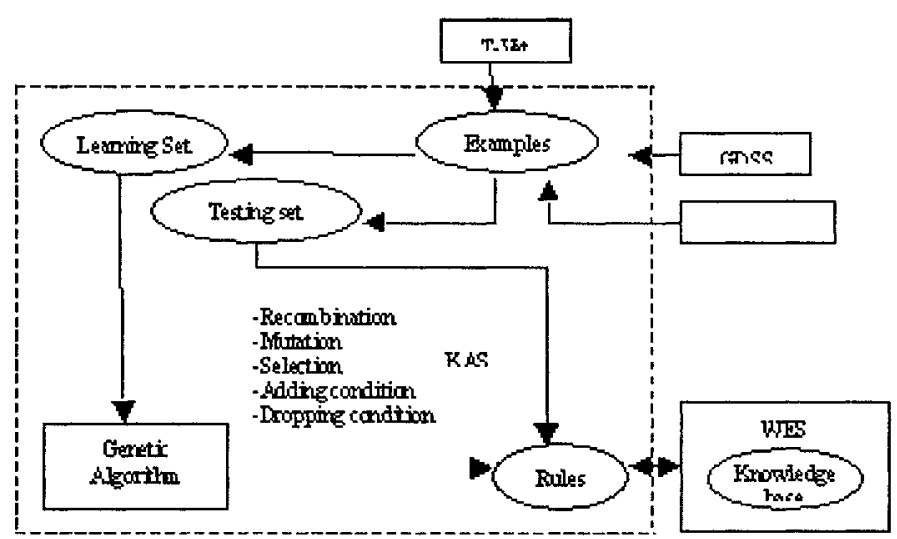

Fig. 2. The Structure of KAS

Case-based reasoning system is a system that solves current problems by adapting to or reusing the used solutions to solve past problems. In case-based reasoning, the study about case adaptation can be divided into two areas. One is about adaptation method and adaptation knowledge that are used at adaptation stage. The other is to reduce the necessity of case adaptation by extracting the most appropriate case for current problems. The former focuses on how to acquire case adaptation knowledge, and the latter focuses on the necessity about case adaptation.

However, the bottleneck phenomenon in acquiring knowledge may be caused in case-based systems. To resolve the bottleneck at the case-based reasoning, a research that automatically acquires knowledge needed for case-based reasoning by using the technologies of machine learning or data mining has been studied (Fig. 2.)

To achieve this intention, we propose a Knowledge Acquisition System (KAS) of adaptation knowledge from derived cases. That is, we construct case base by acquiring cases from Call Center, T-Vet, GDSS automatically acquire adaptation knowledge exploiting data mining concept, and deduce the bottleneck of acquiring adaptation knowledge used at the case adaptation stage of case-based reasoning systems.

\section{The diagnosing process}

There are 4 kinds of users in the H-Vet, such as computer-not-owned fish farmer, computer- owned fish farmer, vet, and call center agent, the work process for all kinds of users can be seen in fig. 3 .

For computer-not-owned fish farmer, he or she can describes the symptoms of their fishes' disease to the call center agent by telephone, and the call center agent inputs all these symptoms into the Fish-Expert interface, and tell the fish farmer the diagnosis result based on the web-based expert system if there is a same disease case in the case base, meantime, this case will be input in the knowledge acquisition system as an successful case. the agent also can ask some questions to the fish farmer based on the web-based expert system, which can add some useful information for fish disease diagnosis. 


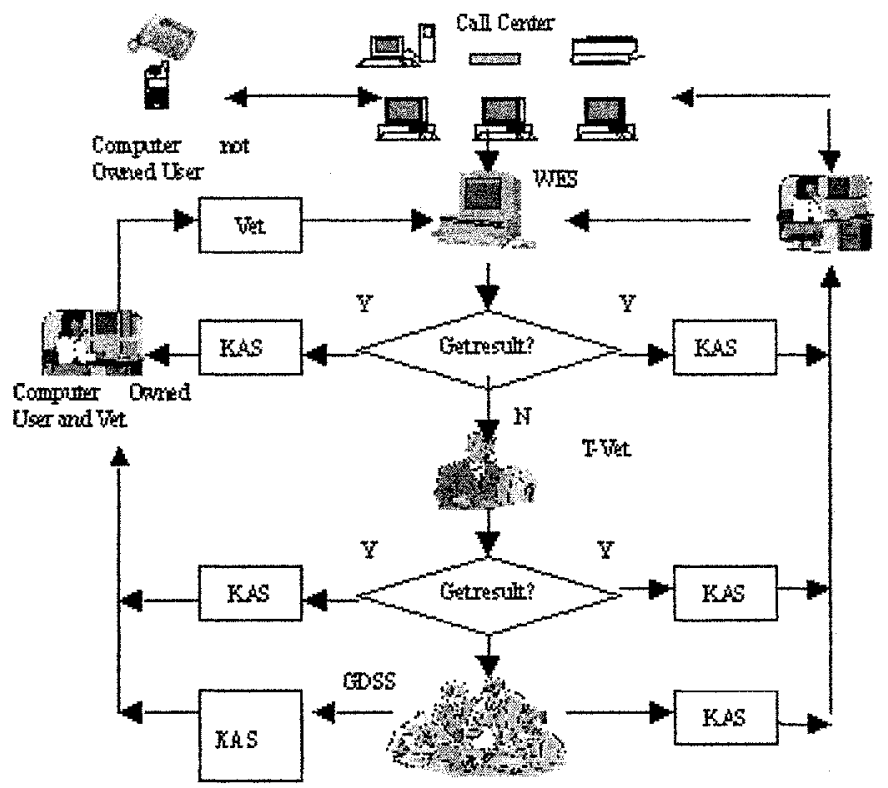

Fig. 3. The work process of $\mathrm{H}-\mathrm{Vet}$

The agent can login on the T-Vet (synchronous tele-diagnosis subsystem) interface get as on line help from the vet, and then tell result to the fish farmer, at the same time, this case will be input into the knowledge acquisition system as an successful case. If the agent can't reply the agent's question, the agent will login the GDSS, and submit the question the GDSS, and reply the fish farmer's question base don the GDSS's diagnosis, at the same time the question and the result will be input the knowledge acquisition system as a successful disease diagnosis case.

For computer-owned fish farmer, he/she can login the web-based expert system directly, and use to the Fish-Expert to solve his or her question, if the Fish-Expert can't answer the farmer's question, the farmer can login T-Vet system and get a online help from vet or get a synchronous tele-diagnosis help, he can login the GDSS system if T-vet can't help him/her. All successful disease diagnosis will be input the knowledge acquisition system as a successful case during the whole work process.

For call center agent, the main task is to wait computer-not-owned fish farmers' call, and answer their questions base on the Fish-Expert, T-vet and GDSS, the interface and work process for agent same as computer-owned fish farmer.

For Vet, the main task is to answer the questions which the expert system can't solved, there are 2 interfaces for them, one is T-Vet, another is GDSS, the participation of the vet will provide many practical disease diagnosis, which will be added to the case base. 


\section{Implementation}

System tests, such as logic tests, debugging, rule checking and sample field tests were carried out by system developers. This was to ensure the system would work correctly before it was distributed to farmers. After the system testing, H-Vet available for pilot implementation in North China, in cities such as Beijing, Tianjin, and Shandong provinces. User feedback was gathered by conducting interviews and collecting information through the system's built-in visitor feedback form. In general, the system has been an effective aid to fish farmers, fishery experts and a reference system to fish vets. Some interfaces are as follows. (Fig. 4.-7)

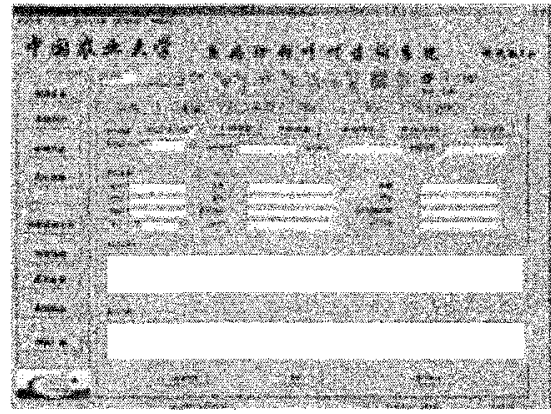

Fig. 4 Interface of Call Center

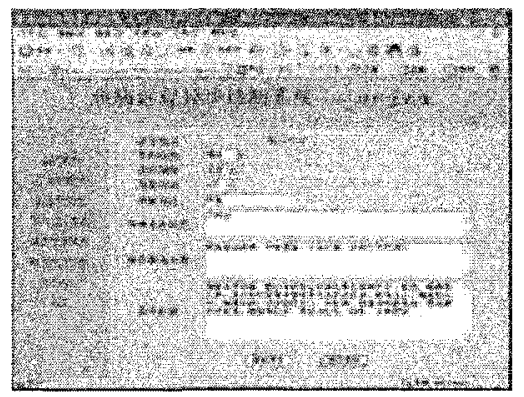

Fig. 6 Interface for Computer Owned User

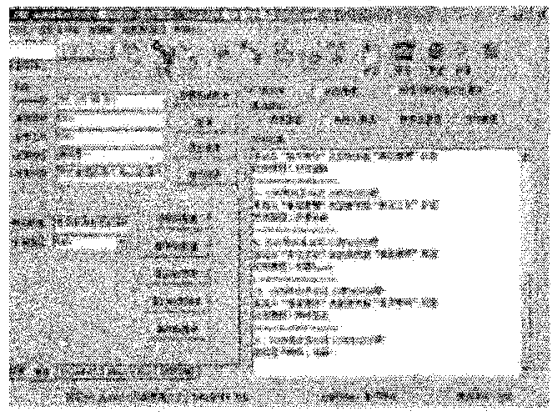

Fig. 5 Interface of Agent

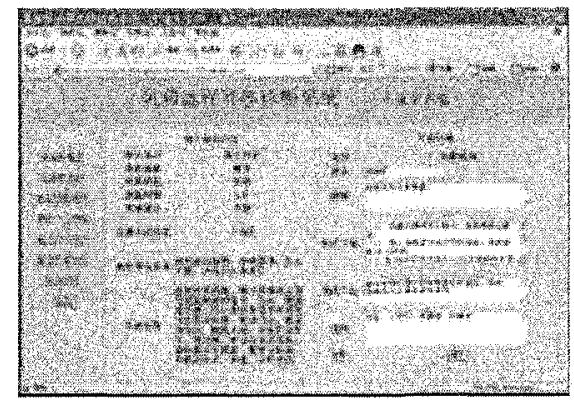

Fig. 7 Interface of T-Vet

\section{Conclusions}

This paper reports a research attempt in developing a hybrid tele-diagnosis system in aquaculture, the system provides a tool for computer and Internet not owned user using web-based expert system, for knowledge automatically acquisition. The research provides some new ideas to solve the bottleneck of traditional expert system.

The wide spread of Internet, Intranet, call center, telecommunication infrastructures and intelligent software will facilitate the work of hybrid telediagnosis system, the frame work proposed in this paper and the specific issues list in section 3 and 4 cover the major topics perceived by the researchers as warrant further 
investigation. As our $\mathrm{H}$-Vet knowledge base increase, more research issues will undoubtedly be added.

The next phase in our research could be the refinement of the framework and testing its value by researchers and practitioners. Also, the identification of software tools that will enhance the multi-site and interorganizational meeting process, and the development of procedures and training methods that will help organizations take advantage of the benefits of using a DGSS need to be researched. The data-mining algorithm will be a key work in the future research, which will play a very important role in knowledge refining and acquisition.

The DGSS environment offers many potential areas for investigation,. The research framework and issues raised in this paper are intended for researchers and practitioners who are interested in looking at the impact of the DGSS environment on fish farmers, vet groups, and call center agents.

\section{Acknowledgement}

The research was funded by the Huo Yingdong foundation in China (project number: 94032). We would like to thank many domain experts from the Beijing Aquaculture Science Institute, Aquaculture Department of Tianjin Agricultural College, Aquaculture Bureau of Shandong province, for their co-operation and support. Our special thanks should also go to Prof. Kezhi Xing, Mr Yongjun Guo at Tianjin Agricultural College for his valuable suggestions and comments on the system.

\section{References}

1. Aksin, O. Z., Harker, P.T.( 2003). Capacity sizing in the presence of a common shared resource: imensioning an inbound call center. European Journal of Operational Research 147, 464 * 483

2. Bendoly, E., Bachrach, D. G., (2003). A process-based model for priority convergence in multi-period group decision-making. European Journal of Operational Research 148 (2003) 534• 545

3. Duan, Y., Fu, Z., Li, D. (2003). Toward developing and using Web-based telediagnosis in aquaculture. Expert System with Applications, 25, 247-254

4. Grove, R. (2000). Internet-based expert systems. Expert Systems, 17(2), 129-135.

5. Guo, Z (2001). Analysis and prospects for China's aquatic market in 2000. www.ifishery.com/jrdd/2001060601.htm

6. Li, D., Fu, Z., \& Duan, Y. (2002). Fish-expert: A web-based expert system for fish disease diagnosis. Expert System with Applications, 23(3), 311-320.

7. Lim, S. S., Pang, Y. K., \& Tan, H. S. (2001). Telemedicine VSATapplications: Temasek polytechnic experience. International Telehealth Symposium, 14 March, Bangkok, United Nations Conference Center.

8. Magrabi, F., Lovell, N. H., \& Cellar, B. G. (1999). A Web-based approach for electrocardiogram monitoring in the home. International Journal of Medical Informatics, 54, 145-153. 
9. Potter, W. D., Deng, X., Li, J., Xu, M., Wei, Y., Lappas, I., Twery, M. J., \& Bennett, D. J. (2000). A Web-based expert system for gypsy moth risk assessment. Computer and Electronics in Agriculture, 27(1-3), 95-105.

10. Power, D. J. (August 10-13 2000). Web-based and model-driven decision support systems: Concepts and issues. Proceedings of Americas Conference on Information Systems (AMCIS 2000), Long Beach, California.

11. Rajani, R., \& Perry, M. (1999). The reality of medical work: The case for a new perspective on telemedicine. Virtual Reality Journal, 4, 243-249.

12. Riva, A., Bellazzi, R., \& Montani, S (1998). A knowledge-based web server as a development environment for Web-based knowledge servers. IEE Colloquium on Web-based Knowledge Servers (Digest No.1998/307).IEE. 1998, 5/1-5. London, UK 13. Sedbrook, T. A. (1998). A collaborative fuzzy expert system for the Web. Data Base for Advances in Information Systems, 29(3), 19-30.

14. Tung, L., Turban, E. (1998). A proposed research framework for distributed group support systems. Decision Support System, 23, 175-188

15. Stohr, E.A., Konzynski, B.R. (1992) Information Systems and Decision Process, IEEE Computer Society Press, Los Alamitos, CA. 